\title{
Determining THE CorRelation Between the TP53 Gene AND Kidney CANCER Survival Through the CoX PROPORTIONAL-HAZARDS REGRESSION MODEL
}

\author{
Rohan Bhansali \\ Loudoun Academy of Science \\ Ashburn, VA 20148 \\ rohanbhansali@connectai.org
}

January 31,2021

\begin{abstract}
Kidney cancer is among the most common and deadly forms of cancer and its incidence has spiked considerably in recent years. It contains a high propensity to rapidly spread to nearby organs, making swift and early diagnosis absolutely critical to ensuring optimal patient recovery. However, its subtle symptoms and manifestation in laboratory tests can make discerning its occurrence difficult. Predicting the probability of the carcinoma based upon various gene mutations has been previously explored, but this study specifically focuses on the TP53 mRNA and the effect its variants have. The Cox proportional-hazards regression technique, ubiquitously regarded as the most accurate method for survival modeling, is utilized to determine that there is a statistically significant difference in the renal cell carcinoma survival of patients with and without the TP53 gene expression. Specifically, possessing this gene, which itself encodes a tumor suppressor protein, correlates with a much higher survival rate from the carcinoma. This finding contains large implications for future exploration within the intersection of genomics and oncology and suggests the efficacy of gene prediction in carcinomas and other pathologies with hereditary predilections.
\end{abstract}




\section{Introduction}

\subsection{Renal Cell Carcinoma}

Renal cell carcinoma, the most common form of kidney cancer, has the highest mortality rate of the genitourinary cancers and has increase in incidence by 500\% since 1971 [9]. Symptoms of afflicted patients can be diverse but generally consist of hematuria, pain, and a flank mass in advanced cases. It originates from uncontrolled cancer cell growth in the inner linings of the kidneys' tubules and often spreads to surrounding organs, including the lungs. Indicators of renal cell carcinoma include abdominal swelling and enlarged veins in the scrotal sac. Standard procedure for doctors to confirm the carcinoma diagnosis entails a complete blood count, CT scan, abdominal and kidney ultrasounds, urine examination, or a biopsy. Classifying the cancer from stage 1 to stage 4 (ordered by ascending severity) is performed through bone scans, PET scans, and chest X-rays [1]. Being an autosomal dominantly inherited disease, renal cell carcinoma can be predicted from an individual's gene expression profile using DNA microarrays.

\section{$1.2 \quad$ TP53 mRNA}

The TP53 gene encodes a tumor suppressor protein that contains oligomerization, DNA binding, and transcriptional activation domains [6]. This protein is capable of responding to a variety of cellular stresses in order to efficiently regulate the expression of target genes that induce changes in metabolism, DNA repair, senescence, apoptosis, and cell cycle arrest. Mutations in this gene tend to correlate with hereditary carcinomas such as Li-Fraumeni syndrome [3].

\subsection{Semiparametric Models}

Non-parametric models are often limited in their ability to model covariate effects while fully parametric models tend to overdo assumptions about the data [7]. As a semiparametric estimator, the Cox model's regression portion makes parametric assumptions regarding the effects predictors have on the hazard function; however, it doesn't make any assumptions concerning the hazard function itself [2].

\subsubsection{Proportional-Hazards Regression}

The proportional-hazards model, more commonly referred to as the Cox model, is one of the most frequently used methods for survival data analysis [4, 5]. It models the incidence or hazard rate, which is the number of new disease cases per population at-risk per unit time.

\section{Methodology}

\subsection{The Cancer Genome Atlas}

The Cancer Genome Atlas (TCGA) is the world's largest and most comprehensive collection of genomic data seeking to empower new discoveries relating to the underlying molecular basis for approximately two hundred different types of carcinomas [8]. Since its inception in 2005, the government funded project has generated nearly three petabytes of genomic, epigenomic, transcriptomic, and proteomic data. The publicly available database was accessed through the Genomic Data Commons Data Portal, supported by additional data processing, visualization, and computational tools.

\subsection{Survival Function}

The data was used to construct a series of survival time distributions.

\subsection{Molecular Variable}

The molecular variable is numeric; expression data often have skewed distributions, which is why gene expression and miRNA expression are transformed using the logarithmic scale.

\subsection{Cox Regression}

The molecular variable was interpreted as numeric in the Cox regression model. 
Table 1: Summary of survival type and patient number

\begin{tabular}{lllllll}
\hline Disease & Survival type & Patients & Time & Status & Time equals zero & Patients with valid data \\
\hline KIRP & disease-free interval & 291 & 107 & 107 & 1 & 183 \\
KIRP & disease-specific survival & 537 & 1 & 4 & 2 & 284 \\
KIRP & overall survival & 291 & 1 & 0 & 2 & 288 \\
KIRP & progression-free interval & 291 & 2 & 0 & 3 & 286 \\
KIRC & disease-free interval & 537 & 420 & 420 & 2 & 115 \\
KIRC & disease-specific survival & 537 & 0 & 12 & 2 & 523 \\
KIRC & overall survival & 537 & 0 & 0 & 2 & 535 \\
KIRC & progression-free interval & 537 & 2 & 0 & 2 & 533 \\
KICH & disease-free interval & 66 & 37 & 37 & 0 & 29 \\
KICH & disease-specific survival & 66 & 1 & 0 & 0 & 65 \\
KICH & overall survival & 66 & 1 & 0 & 0 & 65 \\
KICH & progression-free interval & 66 & 1 & 0 & 0 & 65 \\
\hline
\end{tabular}

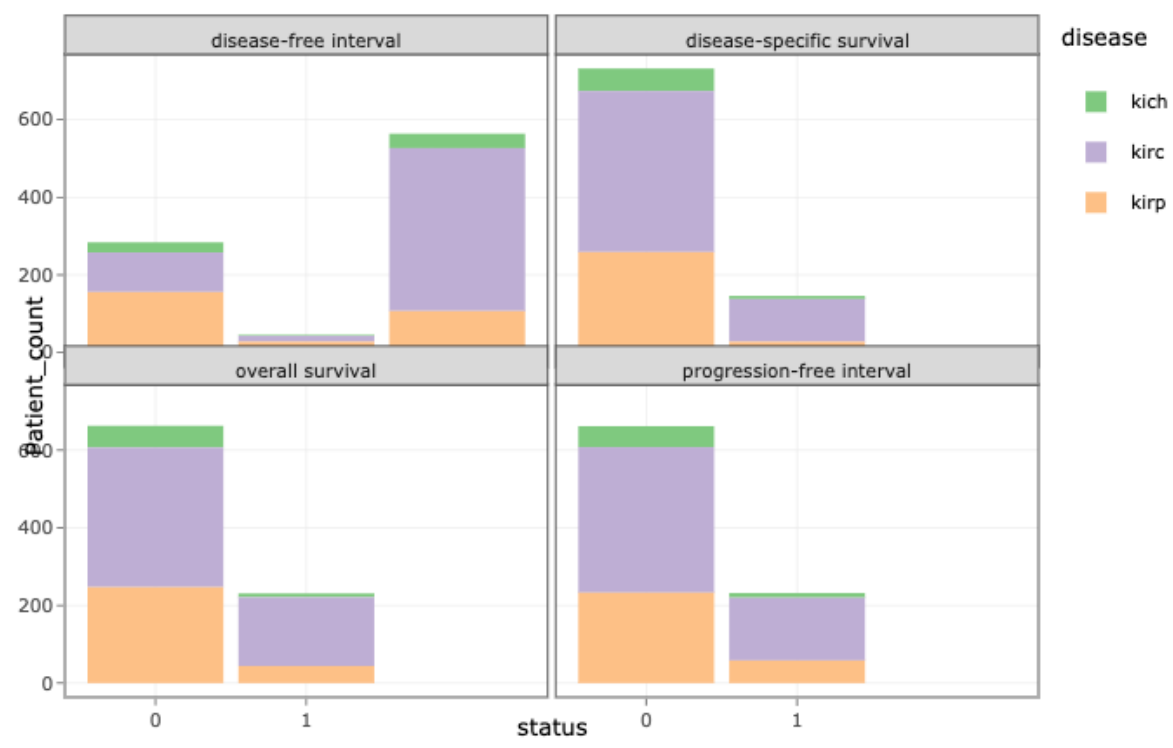

Figure 1: Survival status summary ( 0 denotes alive, 1 denotes death)

\subsection{Kaplain-Meier}

Patients were split into "High" and "Low" groups using the medium value as the cutoff.

\section{Conclusion}

The results of the Cox regression coupled with the Kaplain-Meier plots provide strong indications of a correlation between the presence of the TP53 gene and kidney cancer mortality. The model supported a lower mortality rate for patients possessing the TP53 gene, which likely stems for the gene being an encoder for the tumor suppressor protein responsible for initiating the biochemical reactions that hinder the growth of the carcinoma. This finding is a significant step towards being able to comprehensively screen and detect the occurrence of carcinomas in order to adequately treat them prior to their spread. 


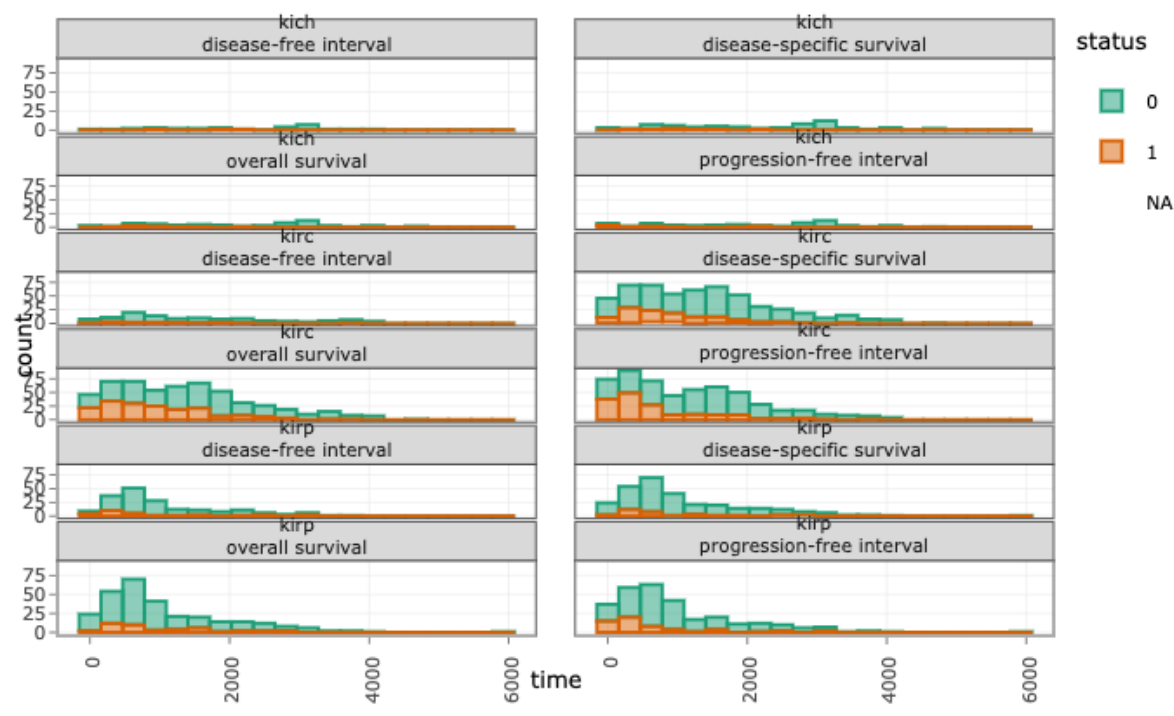

Figure 2: Survival time distribution



Figure 3: Molecular variable summary

\section{References}

[1] Cairns, P. (2012). Renal Cell Carcinoma. Cancer Biomark, 9(1-6), 461-473. doi:10.3233/CBM-2011-0176

[2] Fisher, L. \& Lin, D.Y. (1999). Time-Dependent Covariates in the Cox Proportional-Hazards Regression Model. Annual Review of Public Health, 20(1), 145-157. doi:10.1146/annurev.publhealth.20.1.145

[3] Haronikova, L., Olivares-Illana, V., Wang, L., Karakostis, K., Chen, S., \& Fåhraeus, R. (2019). The p53 mRNA: an integral part of the cellular stress response. Nucleic Acids Research, 47(7), 3257-3271. doi:10.1093/nar/gkz124

[4] Harrell, F. (2015). Cox Proportional Hazards Regression Model. Regression Modeling Strategies, 475-519.

[5] Heinzl, H. \& Kaider, A. (1997). Gaining more flexibility in Cox proportional hazards regression models with cubic spline functions. Computer Methods and Programs in Biomedicine, 54(3), 201-208. doi:10.1016/S0169- 
Table 2: Cox regression

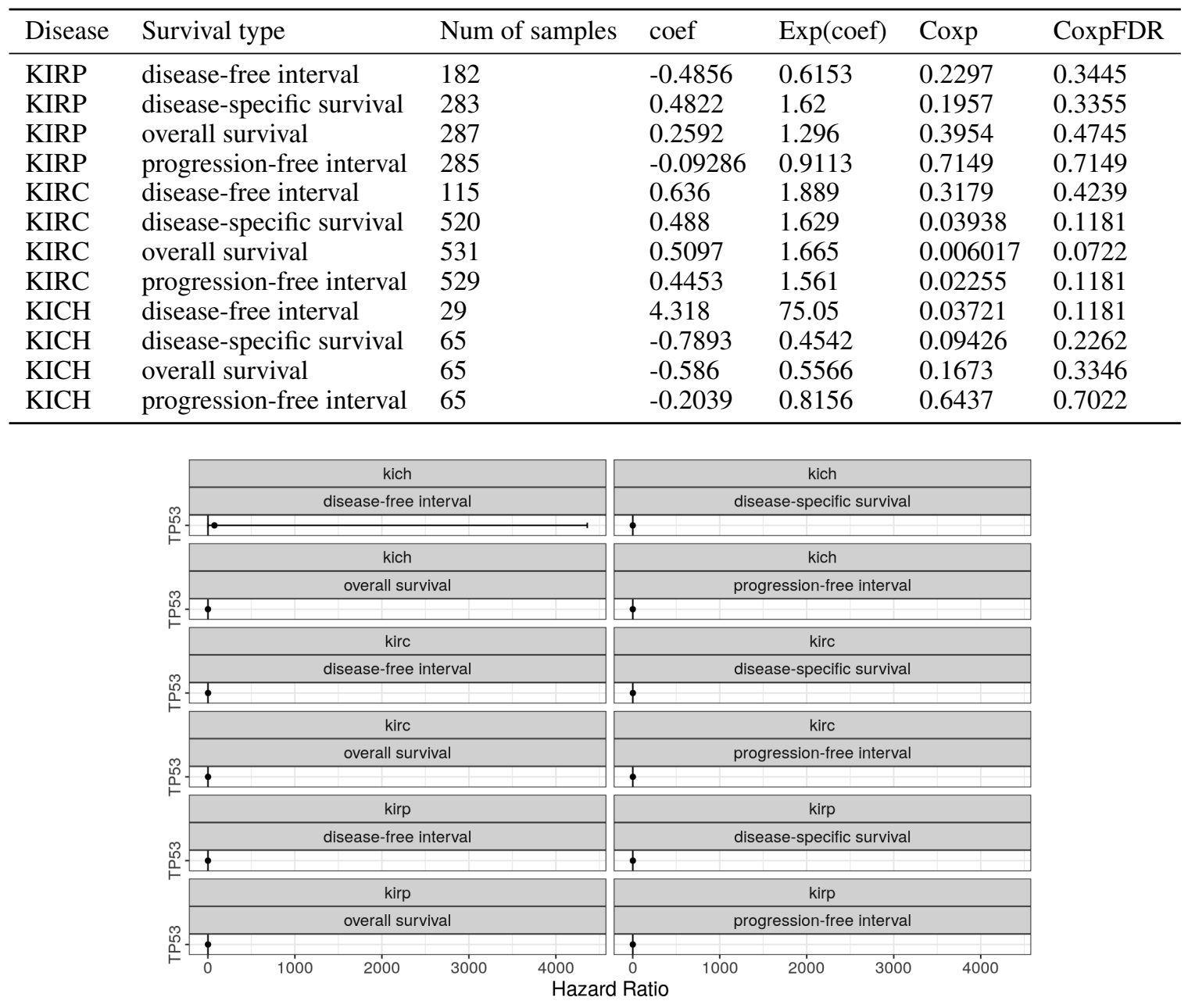

Figure 4: Hazard ratio and 95\% CI

\section{7(97)00043-6}

[6] Karakostis, K. \& Fåhraeus, R. (2019). Shaping the regulation of the p53 mRNA tumour suppressor: the co-evolution of genetic signatures. BMC Cancer, 19(915). doi:10.1186/s12885-019-6118-y

[7] Lin, D.Y. \& Wei, L.J. (2012). The Robust Inference for the Cox Proportional Hazards Model. Journal of the American Statistical Association, 84(408), 1074-1078. doi:10.1080/01621459.1989.10478874

[8] Tomczak, K., Czerwińska, P. \& Wiznerowicz, M. (2015). The Cancer Genome Atlas (TCGA): an immeasurable source of knowledge. Contemporary Oncology, 19(1A), A68-77. doi:10.5114/wo.2014.47136

[9] Vogelzang, N. \& Stadler, W. (1998). Kidney cancer. The Lancet, 352(9141), 1691-1696. doi:10.1016/S01406736(98)01041-1 
Table 3: Kaplan-Meier

\begin{tabular}{lllllll}
\hline Disease & Survival type & Num of samples & Var 1 & Var 1 type & LRp & LRpFDR \\
\hline KIRP & disease-free interval & 182 & TP53 & mRNA & 0.7398 & 0.8296 \\
KIRP & disease-specific survival & 283 & TP53 & mRNA & 0.1034 & 0.2482 \\
KIRP & overall survival & 287 & TP53 & mRNA & 0.03452 & 0.2036 \\
KIRP & progression-free interval & 285 & TP53 & mRNA & 0.8296 & 0.8296 \\
KIRC & disease-free interval & 115 & TP53 & mRNA & 0.8181 & 0.8296 \\
KIRC & disease-specific survival & 520 & TP53 & mRNA & 0.06786 & 0.2036 \\
KIRC & overall survival & 531 & TP53 & mRNA & 0.004817 & 0.0578 \\
KIRC & progression-free interval & 529 & TP53 & mRNA & 0.223 & 0.446 \\
KICH & disease-free interval & 29 & TP53 & mRNA & 0.05266 & 0.2036 \\
KICH & disease-specific survival & 65 & TP53 & mRNA & 0.3116 & 0.5342 \\
KICH & overall survival & 65 & TP53 & mRNA & 0.3847 & 0.577 \\
KICH & progression-free interval & 65 & TP53 & mRNA & 0.6156 & 0.8208 \\
\hline
\end{tabular}

TP53 mRNA with disease-free interval in kirp

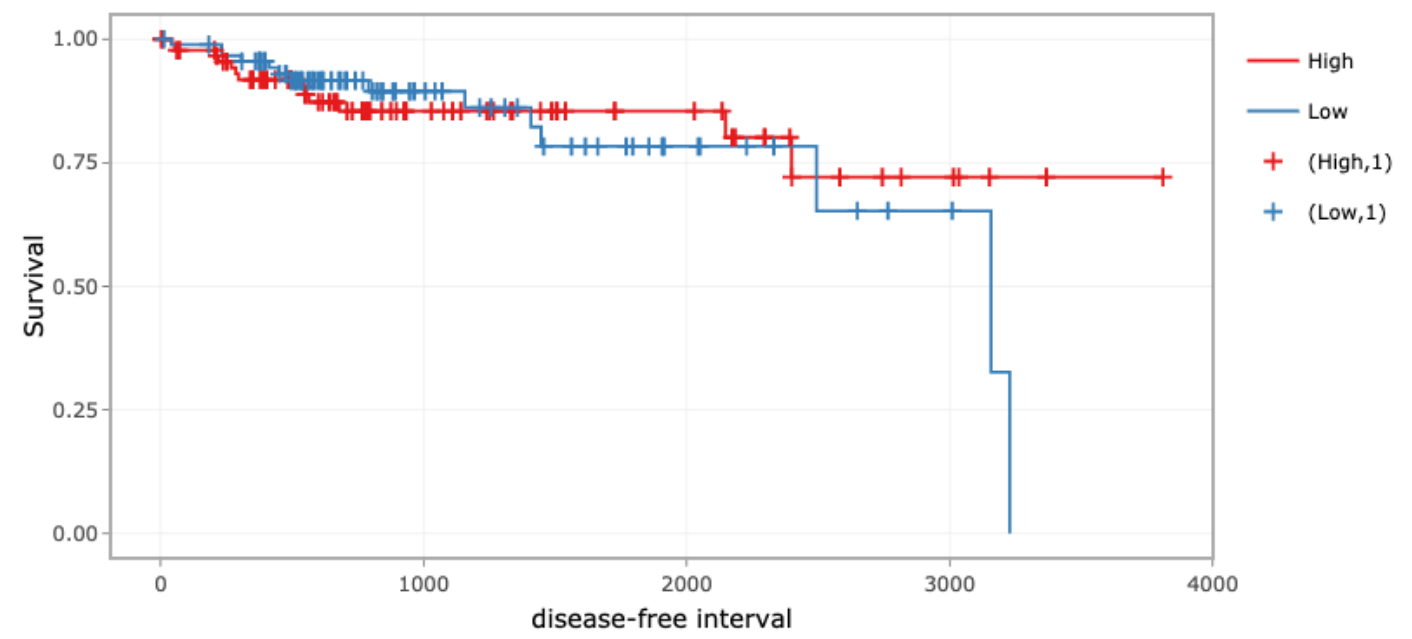

Figure 5: TP53 mRNA with disease-free interval in kirp

TP53 mRNA with disease-specific survival in kirp

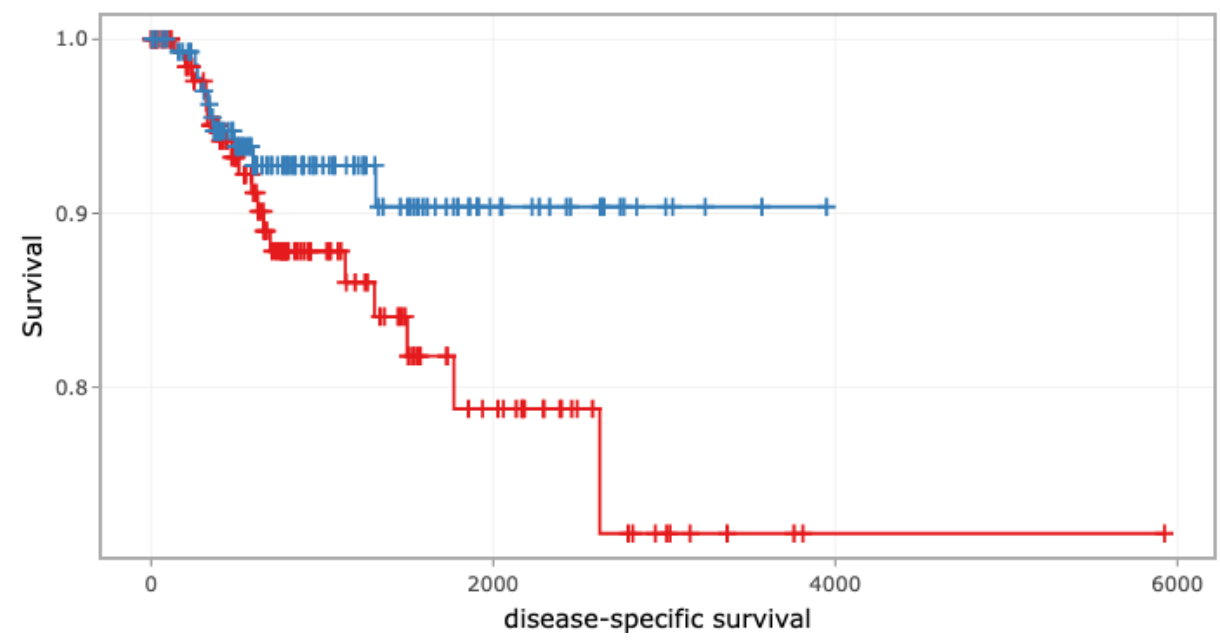

Figure 6: TP53 mRNA with disease-specific survival in kirp

$$
\begin{aligned}
& - \text { High } \\
& - \text { Low } \\
& +\quad(\text { High,1) } \\
& +\quad(\text { Low,1) }
\end{aligned}
$$




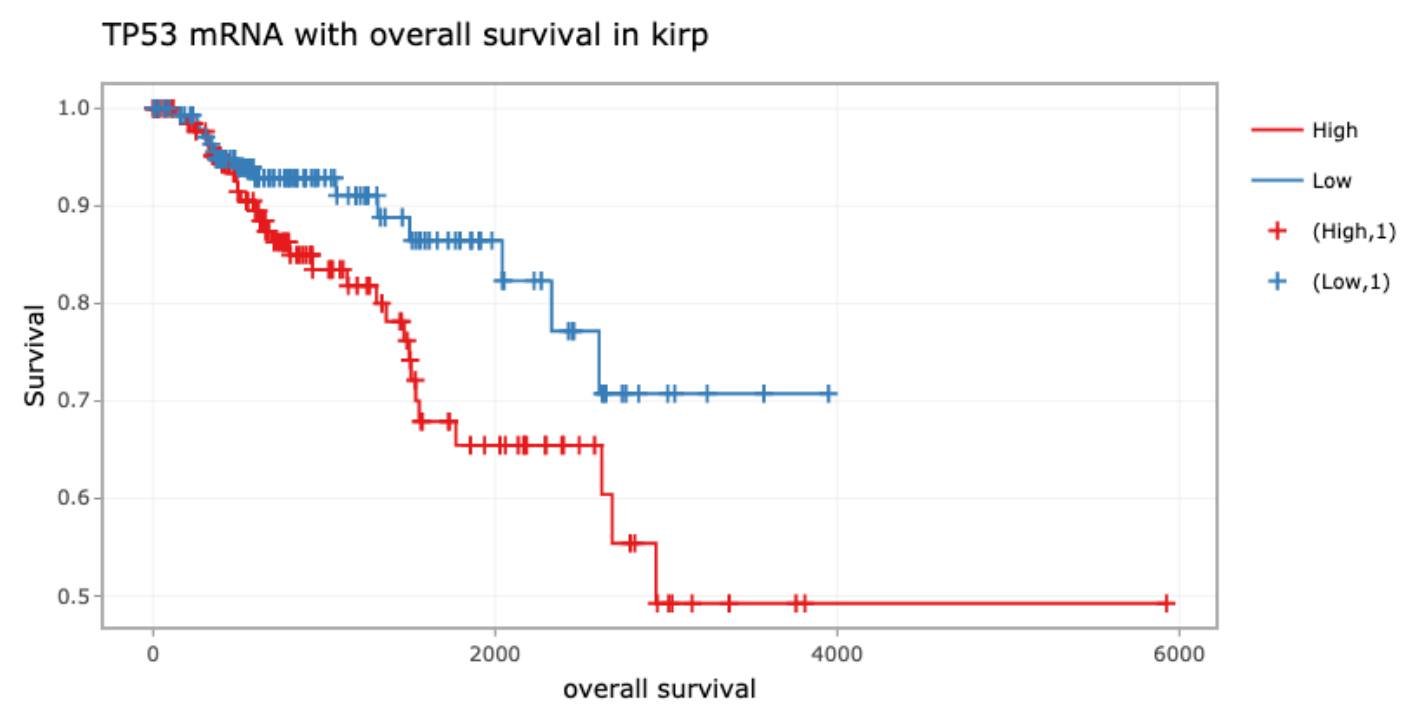

Figure 7: TP53 mRNA with overall survival in kirp

TP53 mRNA with progression-free interval in kirp

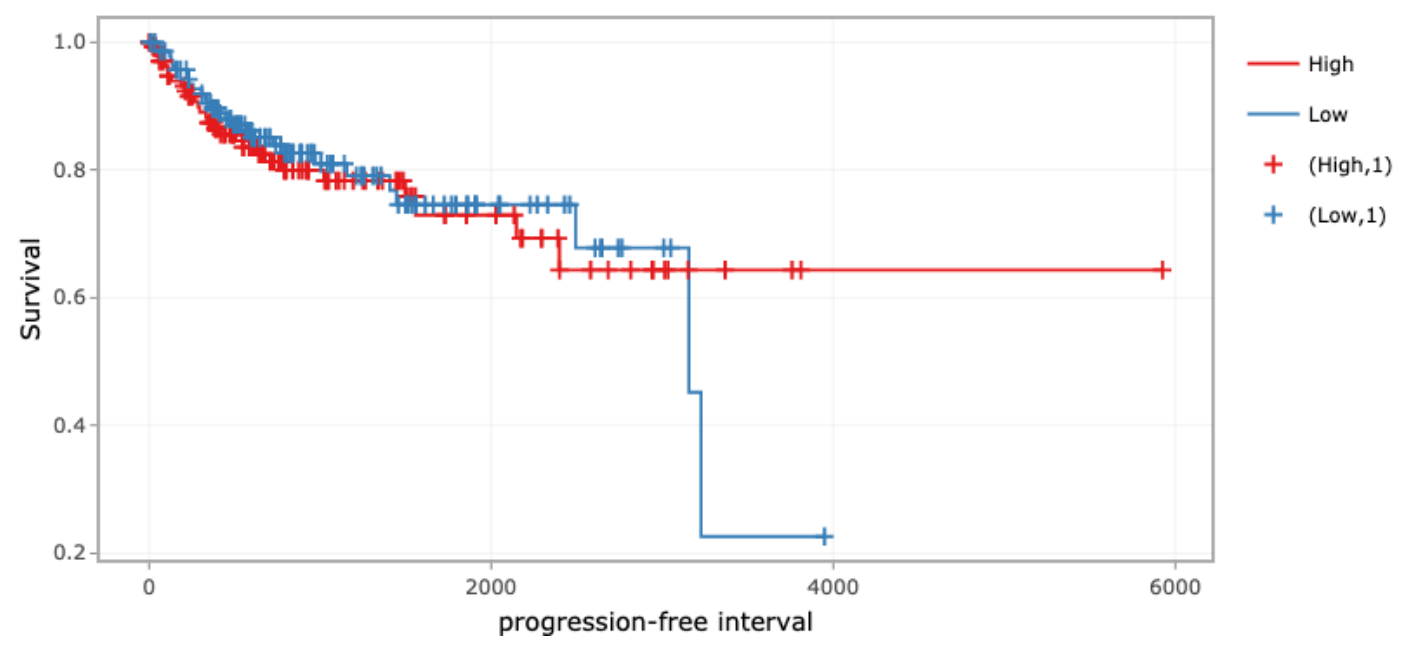

Figure 8: TP53 mRNA with progression-free interval in kirp 


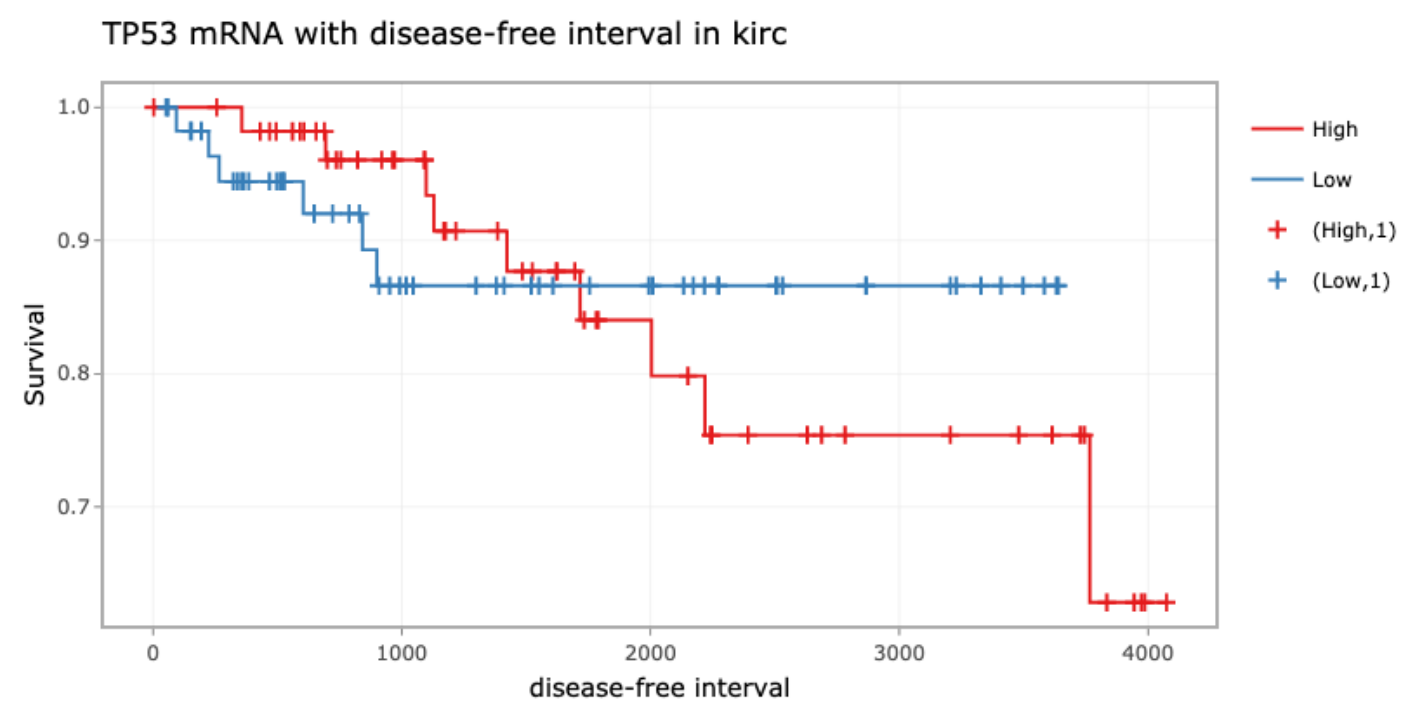

Figure 9: TP53 mRNA with disease-free interval in kirc

TP53 mRNA with disease-specific survival in kirc

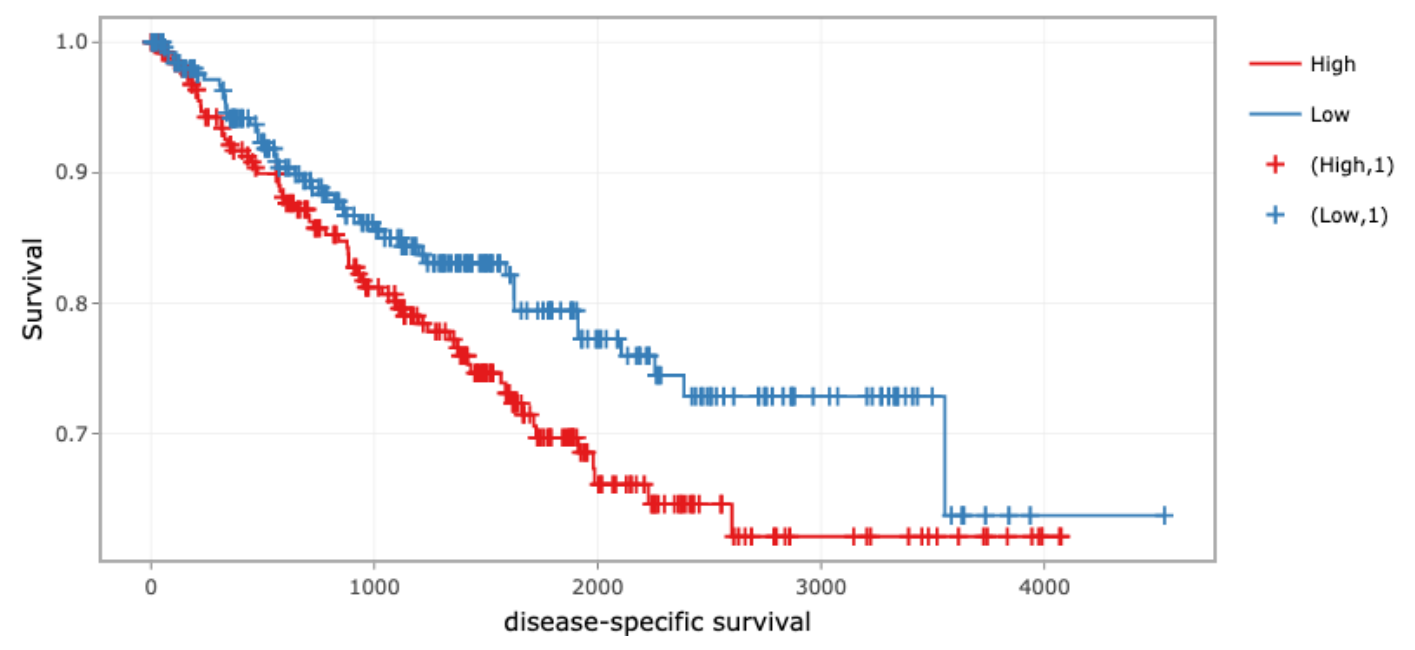

Figure 10: TP53 mRNA with disease-specific survival in kirc 
TP53 mRNA with overall survival in kirc

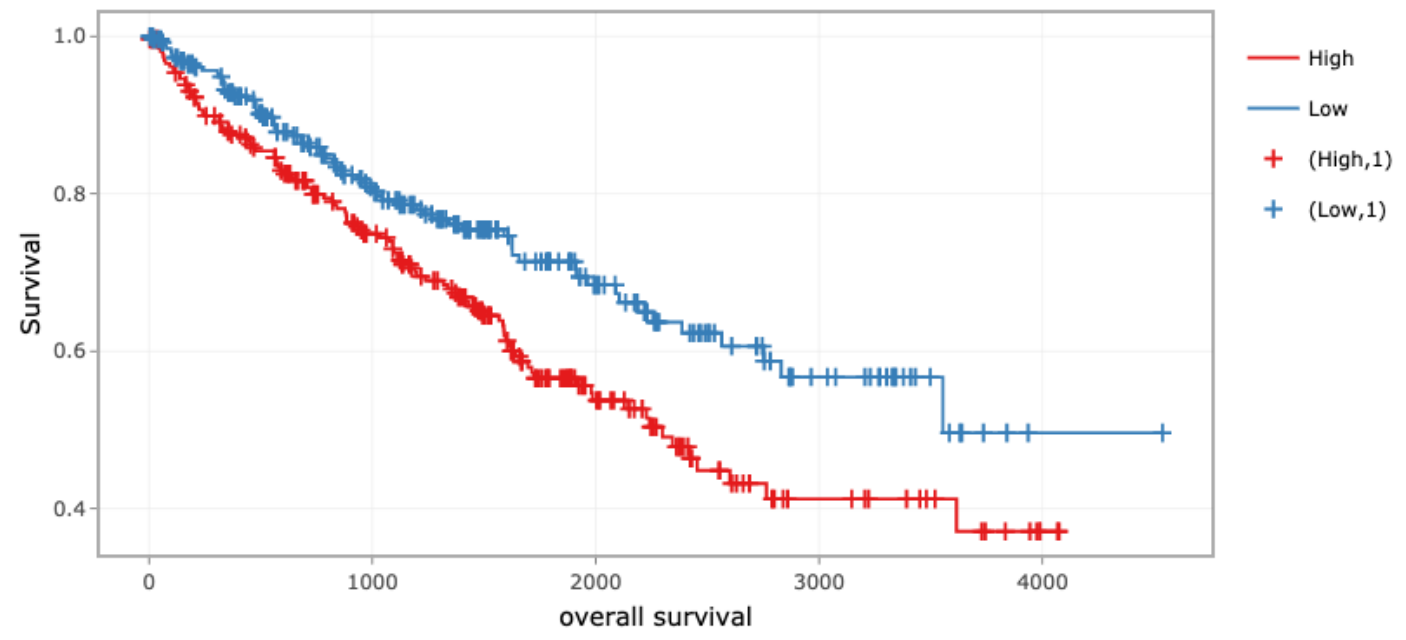

Figure 11: TP53 mRNA with overall survival in kirc

TP53 mRNA with progression-free interval in kirc

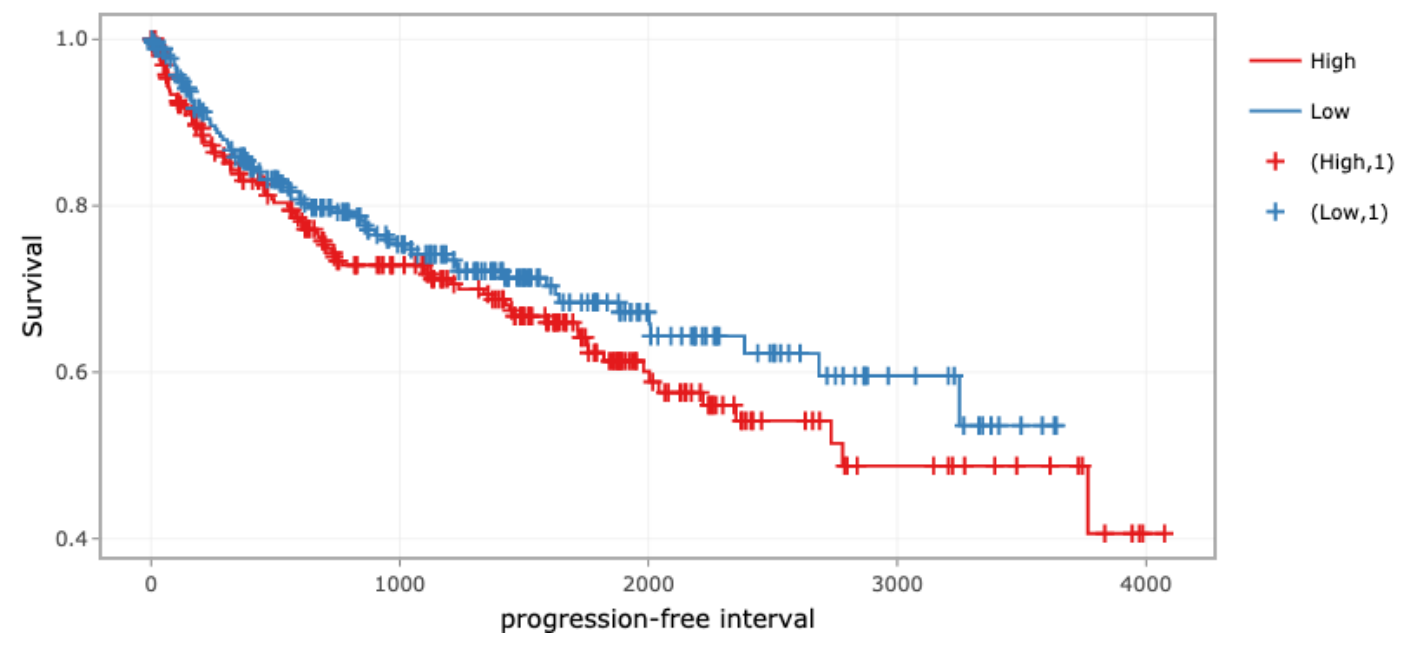

Figure 12: TP53 mRNA with progression-free interval in kirc 
TP53 mRNA with disease-free interval in kich

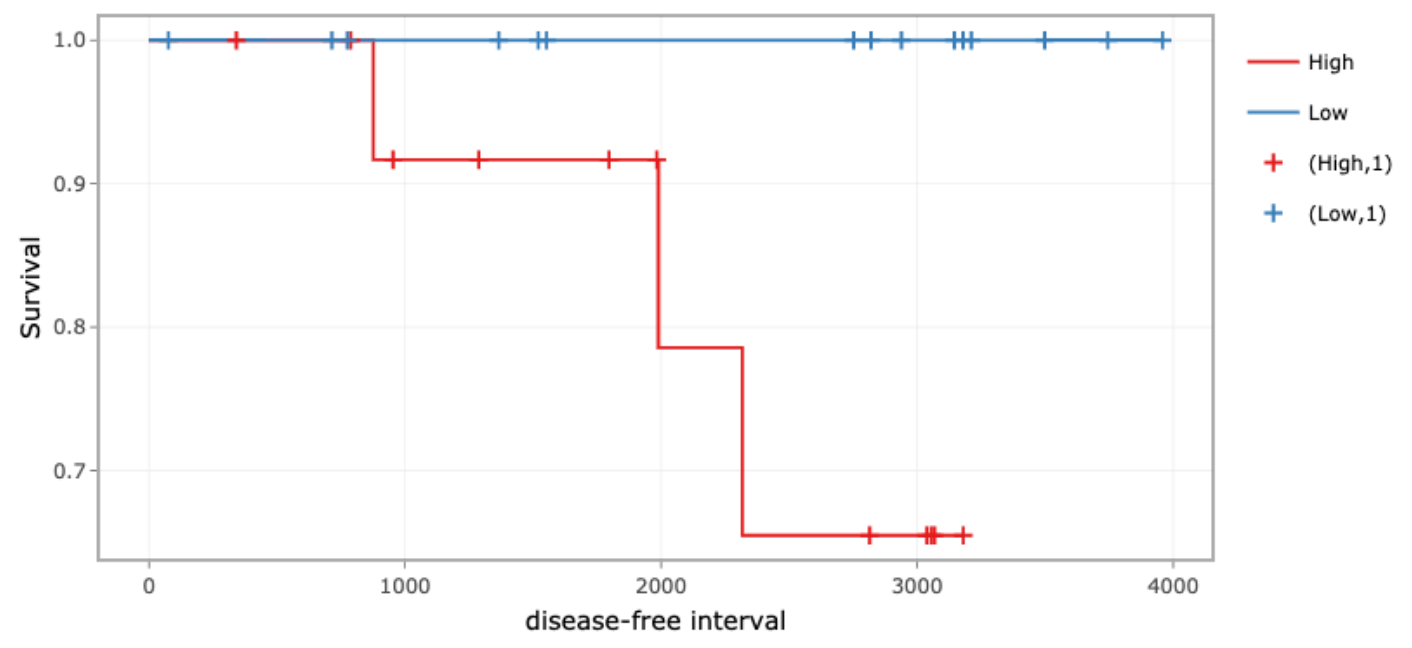

Figure 13: TP53 mRNA with disease-free interval in kich

TP53 mRNA with disease-specific survival in kich

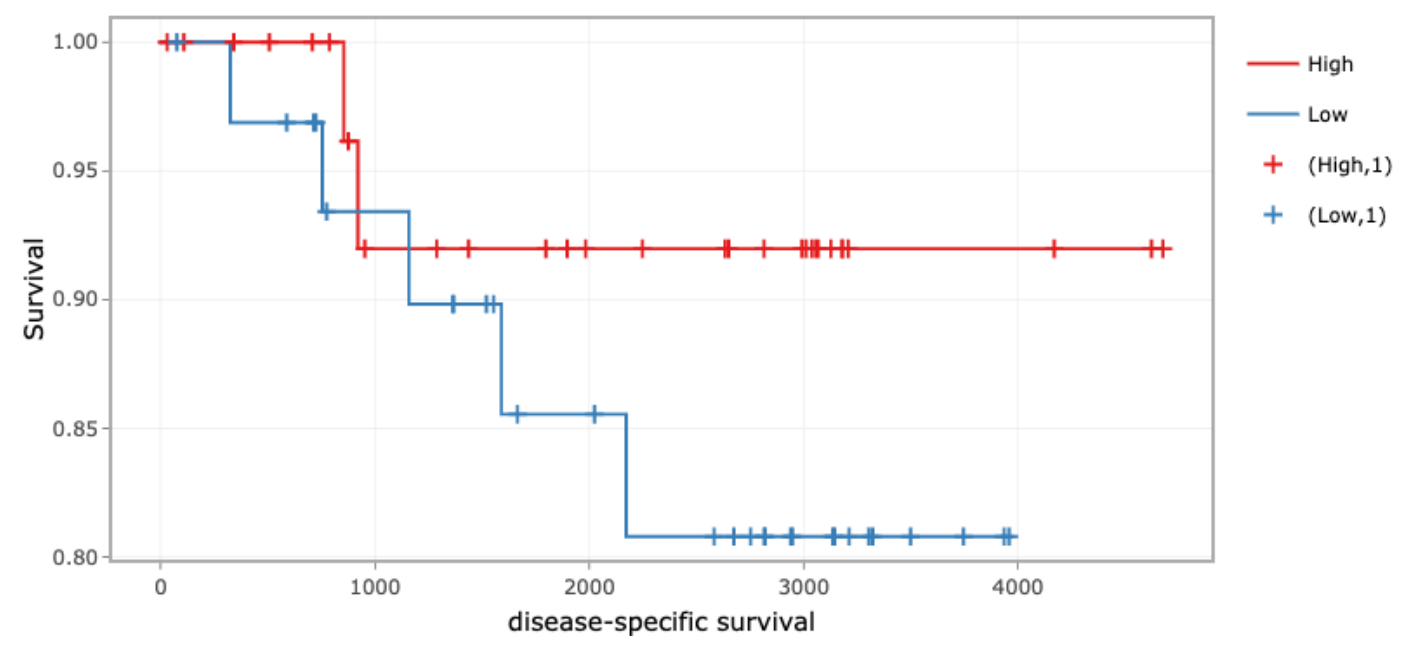

Figure 14: TP53 mRNA with disease-specific survival in kich 
TP53 mRNA with overall survival in kich

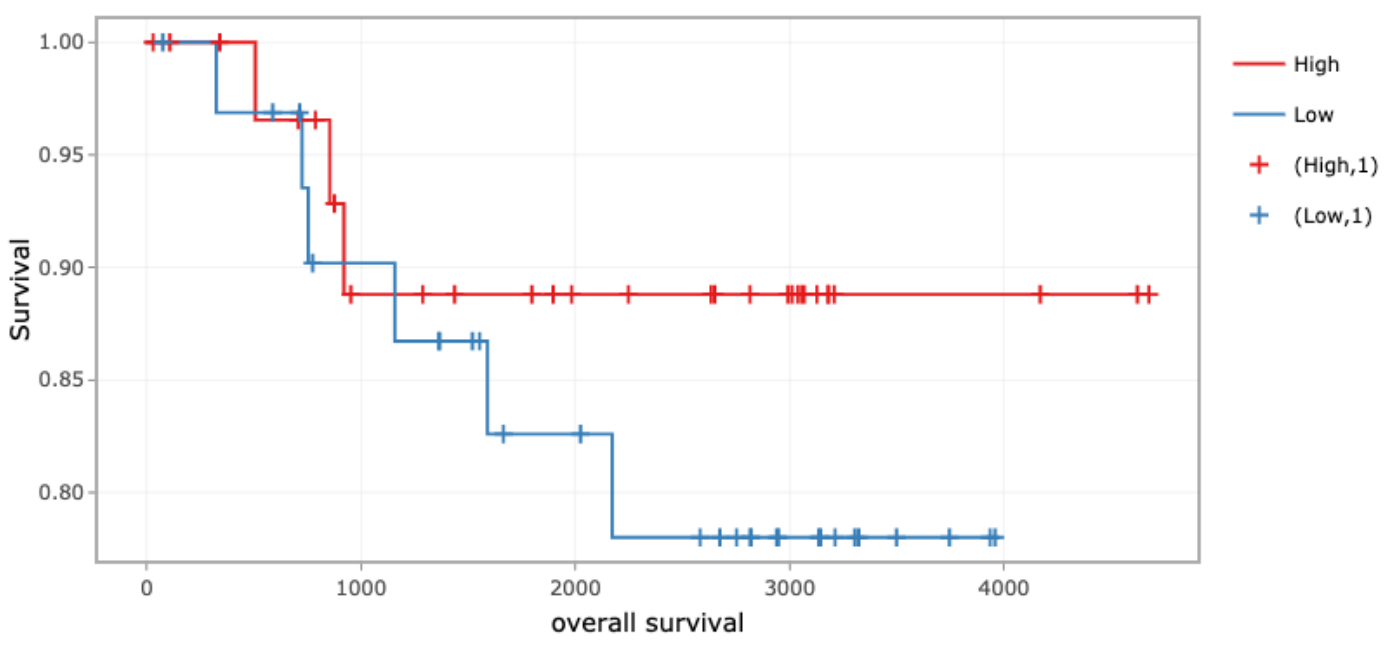

Figure 15: TP53 mRNA with overall survival in kich

TP53 mRNA with progression-free interval in kich

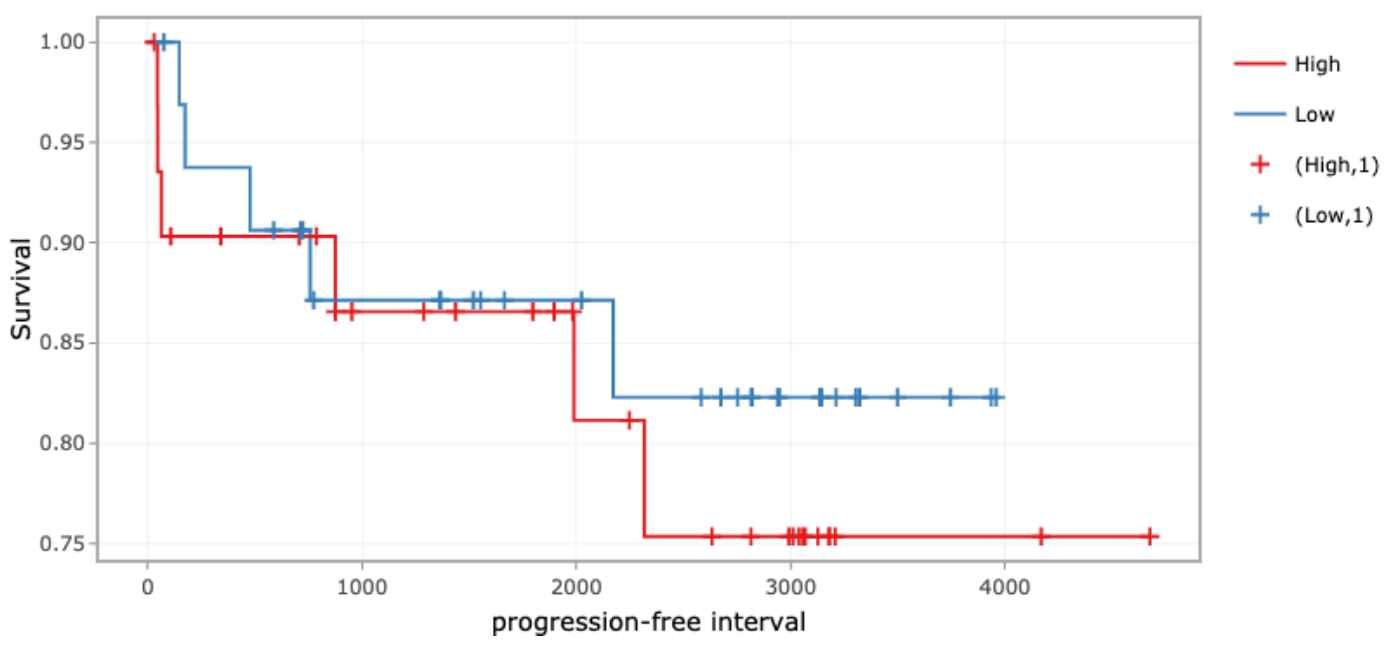

Figure 16: TP53 mRNA with progression-free interval in kich 